\title{
Characteristics Associated with Liver Graft Failure: The Concept of a Donor Risk Index
}

\author{
S. Feng ${ }^{a, *}$, N.P. Goodrich ${ }^{b, c}$, \\ J.L. Bragg-Gresham ${ }^{b, c}$, D.M. Dykstra ${ }^{b, c}$, \\ J.D. Punch ${ }^{d}$, M.A. DebRoy ${ }^{e}$, S.M. Greenstein ${ }^{f}$ \\ and R.M. Merion ${ }^{\mathrm{c}, \mathrm{d}}$ \\ a Department of Surgery, Division of Transplantation, \\ University of California San Francisco, San Francisco, \\ California, USA \\ ${ }^{\mathrm{b}}$ University Renal Research and Education Association, \\ Ann Arbor, Michigan \\ ' Scientific Registry of Transplant Recipients, Ann Arbor, \\ Michigan \\ dDepartment of Surgery, Division of Transplantation, \\ University of Michigan, Ann Arbor, Michigan, USA \\ 'Department of Surgery, Division of Transplantation, \\ University of Medicine and Dentistry of New Jersey, \\ Newark, New Jersey, USA \\ ${ }^{\dagger}$ Department of Surgery, Division of Transplantation, \\ Albert Einstein College of Medicine, Bronx, New York, \\ USA \\ * Corresponding author: Sandy Feng, M.D., Ph.D. \\ fengs@surgery.ucsf.edu
}

Transplant physicians and candidates have become increasingly aware that donor characteristics significantly impact liver transplantation outcomes. Although the qualitative effect of individual donor variables are understood, the quantitative risk associated with combinations of characteristics are unclear. Using national data from 1998 to 2002, we developed a quantitative donor risk index. Cox regression models identified seven donor characteristics that independently predicted significantly increased risk of graft failure. Donor age over 40 years (and particularly over 60 years), donation after cardiac death (DCD), and split/partial grafts were strongly associated with graft failure, while African-American race, less height, cerebrovascular accident and 'other' causes of brain death were more modestly but still significantly associated with graft failure. Grafts with an increased donor risk index have been preferentially transplanted into older candidates ( $>50$ years of age) with moderate disease severity (nonstatus 1 with lower model for end-stage liver disease (MELD) scores) and without hepatitis C. Quantitative assessment of the risk of donor liver graft failure using a donor risk index is useful to inform the process of organ acceptance.

Received 1 August 2005, revised 11 November 2005 and accepted for publication 30 November 2005

\author{
Introduction
}

Increasingly aggressive organ utilization has paralleled the progressive shortage of donor organs (1). In today's desperate climate, the opportunity for transplantation presented by each and every organ of each and every deceased donor is thoroughly evaluated by organ procurement organizations and transplant physicians. As the frontiers of utilization continue to expand previously defined boundaries, there is increasing awareness of the potential impact of aggressive utilization practices on graft and patient outcomes. Analyses that define the effect of specific donor characteristics on the risk of post-transplant graft failure have been performed, particularly in the realm of kidney transplantation. This approach has yielded quantitative descriptions of organ quality, which have lent specificity and granularity to pre-existing qualitative descriptions. Currently, the relative risk (RR) of graft failure for every kidney donor as compared to an 'ideal donor' can be determined based upon published analyses of national data (2). This information, essentially a descriptor of organ quality based solely upon donor characteristics, can facilitate the necessary and important discussions between transplant physicians and candidates at the time of placement on the waiting list and at the time of a specific opportunity for kidney transplantation.

We embarked on the current study with the aim of deriving such descriptors in the setting of liver transplantation. The value of such information is heightened by the lifesaving and life-threatening potential of every decision to either accept or reject a particular opportunity for transplantation. For the potential candidate, the model for endstage liver disease (MELD) scoring system, based solely upon liver transplant candidate characteristics, accurately estimates the risk of death without transplantation (3-5). In discussing transplant possibilities with candidates, quantitation of the risks associated with specific combinations of donor characteristics would be valuable. This study examines putative donor risk factors for graft failure as the foundation for deriving a donor risk index that may facilitate organ acceptance decisions.

\section{Materials and Methods}

\section{Data source}

All information regarding donor characteristics and transplant outcomes were from the Scientific Registry of Transplant Recipients (SRTR), as 


\section{Feng et al.}

submitted by members of the Organ Procurement and Transplantation Network. This study was approved by HRSA's SRTR project officer. HRSA has determined that this study satisfies the criteria for the IRB exemption described in the 'Public Benefit and Service Program' provisions of 45 CFR 46.101(b) (5) and HRSA Circular 03.

Data from 20023 transplants, using livers from deceased donors, performed in the United States between January 1, 1998 and December 31,2002 into adult recipients ( $\geq 18$ years of age) were used to identify factors associated with significantly increased risk of graft loss. Multiple organ transplants were excluded. In order to investigate the utilization of livers from deceased donors with characteristics associated with a higher risk of graft loss, data on 9882 deceased donor livers procured for transplantation between April 1, 2002 and December 31, 2003 were examined.

\begin{abstract}
Analytical methods
Predictors of time to graft failure were identified using Cox regression models. Time to graft failure was defined as the period between transplantation and graft loss secondary to either retransplantation or recipient death, whichever occurred first. All available post-transplant follow-up data were used in the analysis (6). Patients were followed for at least 1 year after transplantation. The median follow-up time was 3 years. Missing values for covariates in the regression model were handled with multiple imputation using standard methods available with SAS version 9.1 (SAS Institute, Cary, $\mathrm{NC}$ ) and IVEWARE imputation software (University of Michigan-Survey Research Center, Ann Arbor, MI) (7). Donor parameters investigated include age; sex; race; ethnicity; height; weight; body mass index; cause of death (COD) (trauma, cerebrovascular accident (CVA), anoxia and other); serum levels of creatinine, blood urea nitrogen, glutamic oxaloacetic and pyruvic transaminases (SGOT [AST] and SGPT [ALT]) and total bilirubin; history of insulin-dependent diabetes mellitus, hypertension, cancer, cigarette, alcohol, and intravenous drug use; cytomegalovirus status; hepatitis B core antibody status; hepatitis $C$ virus antibody status; serum sodium $>170 \mathrm{mEq} / \mathrm{L}$; requirement for inotropic agents (dopamine/dobutamine); cardiac arrest after brain death; administration of anti-convulsants, anti-hypertensive and vasodilators within $24 \mathrm{~h}$ of donor aortic cross-clamp; donation after cardiac death (DCD) and a split/partial liver graft. The parameter estimates for donor factors that were identified as significantly associated with increased risk of graft loss were combined as the foundation for calculating the donor risk index. The donor risk index can be used to compare the RR of graft loss for an organ with a specific set of donor and transplant characteristics to a reference case.
\end{abstract}

To isolate the impact of donor characteristics on liver allograft outcomes, all models were adjusted for recipient and transplant factors that may impact allograft failure. Recipient factors included in all models were age; sex; race; ethnicity; body mass index; underlying liver disease diagnosis (acute hepatic necrosis, cholestatic liver disease, noncholestatic liver disease, metabolic liver disease, malignancy, or other); hepatitis B status; hepatitis C status; cytomegalovirus status; history of previous liver transplant; history of previous abdominal surgery; angina pectoris; diabetes mellitus; cerebrovascular disease; transfusion at time of listing; SGOT (AST); total bilirubin, albumin and creatinine; dialysis status at time of transplantation; medical condition (intensive care unit, in hospital, or out of hospital); Status 1 medical urgency designation; requirement for life support; grade III or IV hepatic encephalopathy; requirement of inotropic support; portal vein thrombosis at time of transplantation and incidental tumor identified during transplantation. Transplant parameters included in all models were ABO compatibility, cold ischemia time and origin of the donor organ beyond the recipient's listing organ procurement organization (shared organ). All analyses were performed using SAS version 9.1.
Table 1: Descriptive statistics for selected donor parameters ( $N=$ 20 023; 1998-2002)

\begin{tabular}{lll}
\hline Donor factor & Frequency & Percent \\
\hline Age & 2394 & \\
$0-17$ & 7852 & 12.0 \\
$18-39$ & 3752 & 39.2 \\
$40-49$ & 3273 & 18.7 \\
$50-59$ & 1896 & 16.3 \\
$60-69$ & 856 & 9.5 \\
70+ & 8112 & 4.3 \\
Female & & 40.5 \\
Race & 2337 & \\
African American & 17143 & 11.7 \\
White & 523 & 85.6 \\
Other & & 2.6 \\
Cause of death & 8921 & \\
Trauma & 8736 & 44.6 \\
Stroke & 1720 & 43.6 \\
Anoxia & 603 & 8.6 \\
Other & 395 & 3.0 \\
Partial/Split & 223 & 2.0 \\
Donation after cardiac death & 988 & 1.1 \\
HBcAb positive & 360 & 4.9 \\
HCV positive & 522 & 1.8 \\
Sodium $>$ 170 mEq/L & Mean & 2.6 \\
& 171.34 & SD \\
Height (cm) & & 12.4 \\
\hline
\end{tabular}

\section{Results}

\section{Donor and recipient characteristics}

The distribution of donor and recipient characteristics of interest are displayed in Tables 1 and 2. Approximately 30\% of transplants were performed using livers from donors greater than 50 years of age. Only $2 \%$ were performed using split or partial livers and $1.1 \%$ used DCD livers. The vast majority of donors (88.2\%) died of either trauma or stroke. Less than $5 \%$ of donors were positive for hepatitis B core antibody and less than $2 \%$ were positive for hepatitis C antibody. Over one-third of transplant recipients (39.2\%) tested positive for hepatitis C and nearly two-thirds (64.4\%) were not hospitalized immediately prior to transplantation.

\section{Risk factors for graft failure}

Seven donor characteristics identified as significantly associated with liver allograft failure are listed in Table 3. These included three donor demographic characteristics (age, race and height), three relating to cause and type of donor death (COD CVA, COD other and DCD) and a split/partial graft. Compared to a reference group of donors $<40$ years of age, increasing age was associated with a significant monotonic increase in the risk of graft failure. Donor age over 60 years was the strongest risk factor for graft failure (RR was 1.53 and 1.65, for donor age 61-70 and $>70$, respectively; both $p<0.0001)$. Livers from AfricanAmerican donors had a 19\% higher risk of graft failure compared to those from white donors (RR 1.19; $p<0.0001$ ). 
Table 2: Descriptive statistics for selected recipient and transplant parameters ( $N=20$ 023; 1998-2002)

\begin{tabular}{|c|c|c|}
\hline Recipient factor & Frequency & Percent \\
\hline \multicolumn{3}{|l|}{$\overline{A g e}$} \\
\hline $18-24$ & 473 & 2.4 \\
\hline $25-34$ & 887 & 4.4 \\
\hline $35-44$ & 3364 & 16.8 \\
\hline $45-54$ & 8345 & 41.7 \\
\hline $55-64$ & 5364 & 26.8 \\
\hline $65+$ & 1590 & 7.9 \\
\hline Female & 7114 & 35.5 \\
\hline \multicolumn{3}{|l|}{ Race } \\
\hline African American & 1536 & 7.7 \\
\hline Other & 1201 & 6.0 \\
\hline White & 17286 & 86.3 \\
\hline \multicolumn{3}{|l|}{ Diagnosis } \\
\hline Fulminant hepatic failure & 1611 & 8.0 \\
\hline Non-cholestatic liver disease & 14039 & 70.1 \\
\hline Cholestatic liver disease & 2291 & 11.4 \\
\hline Metabolic disease & 520 & 2.6 \\
\hline Malignant neoplasm & 613 & 3.1 \\
\hline Other & 949 & 4.7 \\
\hline Hepatitis C positive & 7850 & 39.2 \\
\hline \multicolumn{3}{|l|}{ Medical condition } \\
\hline In ICU & 4370 & 21.8 \\
\hline Hospitalized not in ICU & 2761 & 13.8 \\
\hline Not hospitalized & 12892 & 64.4 \\
\hline Angina & 464 & 2.3 \\
\hline Cerebrovascular disease & 103 & 0.5 \\
\hline Previous transfusion & 6979 & 34.9 \\
\hline Status 1 & 1660 & 8.3 \\
\hline On life support & 1883 & 9.4 \\
\hline Previous liver transplant & 1763 & 8.8 \\
\hline CMV positive & 11670 & 58.3 \\
\hline Pretransplant malignancy & 745 & 3.7 \\
\hline Tumor found at transplant & 769 & 3.8 \\
\hline Previous abdominal surgery & 7365 & 36.8 \\
\hline Inotropes for BP support & 1087 & 5.4 \\
\hline On dialysis & 914 & 4.6 \\
\hline Portal vein thrombosis & 521 & 2.6 \\
\hline Grade III or IV encephalopathy & 5665 & 28.3 \\
\hline \multirow{2}{*}{ Hepatitis B positive } & 1390 & 6.9 \\
\hline & Mean & SD \\
\hline Total bilirubin & 7.09 & 9.83 \\
\hline Serum creatinine & 1.31 & 1.08 \\
\hline Serum albumin & 2.84 & 0.77 \\
\hline BMI & 28.11 & 11.51 \\
\hline SGOT/AST & 269.87 & 1000.90 \\
\hline Transplant factor & Frequency & Percent \\
\hline \multicolumn{3}{|l|}{$\overline{\mathrm{ABO} \text { compatibility }}$} \\
\hline Identical & 18136 & 90.6 \\
\hline Compatible & 1674 & 8.4 \\
\hline Incompatible & 213 & 1.1 \\
\hline \multicolumn{3}{|l|}{ Sharing } \\
\hline Local & 14668 & 73.3 \\
\hline Regional & 4251 & 21.2 \\
\hline \multirow[t]{2}{*}{ National } & 1104 & 5.5 \\
\hline & Mean & SD \\
\hline Cold ischemia time & 8.22 & 3.80 \\
\hline
\end{tabular}

Table 3: Donor factors significantly associated with liver allograft failure $(1998-2002)^{*}$

\begin{tabular}{llll}
\hline Donor parameter & RR & $95 \% \mathrm{Cl}$ & p-Value \\
\hline Age & & & \\
$\quad<40$ & 1.00 & & \\
$40-49$ & 1.17 & $1.08-1.26$ & 0.0002 \\
$50-59$ & 1.32 & $1.21-1.43$ & $<0.0001$ \\
60-69 & 1.53 & $1.39-1.68$ & $<0.0001$ \\
$\quad 70$ & 1.65 & $1.46-1.87$ & $<0.0001$ \\
African-American race (vs White) & 1.19 & $1.10-1.29$ & $<0.0001$ \\
Donor height (per 10 cm decrease) & 1.07 & $1.04-1.09$ & $<0.0001$ \\
COD = CVA & 1.16 & $1.08-1.24$ & $<0.0001$ \\
COD = Other ${ }^{\dagger}$ & 1.20 & $1.03-1.40$ & 0.018 \\
DCD & 1.51 & $1.19-1.91$ & 0.0006 \\
Partial/Split & 1.52 & $1.27-1.83$ & $<0.0001$ \\
\hline
\end{tabular}

*Model also adjusted for donor sex, serum sodium $>170 \mathrm{mEq} / \mathrm{L}$, and $\mathrm{HBcAb}$ status; recipient age, sex, race, ethnicity, BMI, hepatitis B status, hepatitis C status, CMV status, previous liver transplant, previous abdominal surgery, angina, diabetes, cerebrovascular disease, transfusion at time of listing, SGOT, total bilirubin, albumin, creatinine, dialysis status at time of transplantation, medical condition, Status 1, life support, grade III or IV encephalopathy, inotropic support, portal vein thrombosis, incidental tumor identified during transplantation, ABO compatibility, cold ischemia time, and regional or national sharing.

tCause of death was not trauma, stroke, or anoxia.

Although two parameters reflecting donor size were assessed, the association of height was stronger than, and independent of, the association of weight. Compared to trauma as a cause of death, CVA and other causes of death (not trauma, CVA or anoxia) were associated with $16 \%$ and $20 \%$ higher risks of graft failure, respectively (both $p<$ 0.02). A test of the statistical interaction between donor age and CVA cause of death was not significant. DCD status and split/partial grafts were associated with a $51 \%$ and $52 \%$ higher risk of graft failure $(p<0.001)$.

Two transplant factors, cold ischemia time and sharing outside of the local donor service area, were also found to be significantly associated with increased risk of graft loss. These factors were independent; there was no significant interaction between cold ischemia time and donor location. Each additional hour of cold ischemia time was associated with an additional $1 \%$ increased risk of graft loss ( $p=0.008$ ). Compared to grafts transplanted within the local area, grafts that were transplanted outside the local area but within the same region had an $11 \%$ increase in risk of graft loss $(p=0.002)$, while grafts that were transplanted beyond the region (nationally shared) had a $28 \%$ increased risk of graft loss $(p<0.0001)$.

\section{Transplants according to donor risk index}

To facilitate the understanding of the risks associated with combinations of donor factors alone, Table 4 shows the range of donor risk index associated with specific combinations of donor risk factors, the number of transplants 
Table 4: Calculated donor risk index and 1-year graft survival and $95 \%$ confidence interval estimates for specified donor profiles (1998-2002)

\begin{tabular}{|c|c|c|c|}
\hline $\begin{array}{l}\text { Donor } \\
\text { profile }\end{array}$ & $\mathrm{N}(\%)$ & $\begin{array}{l}\text { Range of } \\
\text { calculated } \\
\text { DRI }\end{array}$ & $\begin{array}{l}95 \% \mathrm{Cl} \text { for } \\
\text { adjusted } 1 \text { year } \\
\text { survival estimates }\end{array}$ \\
\hline \multicolumn{4}{|l|}{ None } \\
\hline Under 40 & $6814(34.0 \%)$ & Ref. & $84.8-86.4$ \\
\hline $40-49$ & $1174(5.9 \%)$ & 1.17 & $82.9-86.9$ \\
\hline $50-59$ & $653(3.3 \%)$ & 1.32 & $79.0-84.8$ \\
\hline $60-69$ & $299(1.5 \%)$ & 1.53 & $77.7-85.9$ \\
\hline $70+$ & $140(0.7 \%)$ & 1.65 & $61.3-76.3$ \\
\hline \multicolumn{4}{|c|}{ Cod-Other or Cod-Stroke or Black } \\
\hline Under 40 & $2683(13.4 \%)$ & $1.16-1.20$ & $81.8-84.6$ \\
\hline $40-49$ & $2128(10.6 \%)$ & $1.35-1.40$ & $79.8-83.1$ \\
\hline $50-59$ & $2293(11.5 \%)$ & $1.52-1.58$ & $77.2-80.5$ \\
\hline $60-69$ & $1445(7.2 \%)$ & $1.77-1.84$ & $73.4-77.8$ \\
\hline $70+$ & $655(3.3 \%)$ & $1.91-1.99$ & $72.4-78.7$ \\
\hline \multicolumn{4}{|c|}{ (Cod-Other + Race-Black) or (Cod-Stroke + Race-Black) } \\
\hline Under 40 & $276(1.4 \%)$ & $1.38-1.43$ & $71.7-81.3$ \\
\hline $40-49$ & $365(1.8 \%)$ & $1.61-1.67$ & $73.7-82.0$ \\
\hline $50-59$ & $290(1.5 \%)$ & $1.81-1.89$ & $73.7-82.8$ \\
\hline $60-69$ & $132(0.7 \%)$ & $2.11-2.19$ & $63.4-78.4$ \\
\hline $70+$ & $58(<0.5 \%)$ & $2.27-2.37$ & $71.6-91.0$ \\
\hline \multicolumn{4}{|c|}{ DCD or partial/split } \\
\hline Under 40 & $344(1.7 \%)$ & $1.51-1.52$ & $75.4-83.8$ \\
\hline 40-49 & $34(<0.5 \%)$ & $1.76-1.78$ & $45.6-78.0$ \\
\hline $50-59$ & $17(<0.5 \%)$ & $1.98-2.01$ & $73.0-100.0$ \\
\hline $60-69$ & $6(<0.5 \%)$ & $2.30-2.33$ & $33.3-100.0$ \\
\hline $70+$ & $3(<0.5 \%)$ & $2.49-2.52$ & 23.9-100.0 \\
\hline \multicolumn{4}{|c|}{ (DCD or partial/split) + at least one other factor } \\
\hline Under 40 & $129(0.6 \%)$ & $1.74-3.30$ & $64.9-80.5$ \\
\hline $40-49$ & $51(<0.5 \%)$ & $2.03-3.85$ & $60.7-84.7$ \\
\hline $50-59$ & $20(<0.5 \%)$ & $2.29-4.34$ & $49.0-91.5$ \\
\hline $60-69$ & $14(<0.5 \%)$ & $2.66-5.04$ & $46.4-97.0$ \\
\hline $70+$ & $0(0.0 \%)$ & $2.88-5.45$ & $\mathrm{~N} / \mathrm{A}$ \\
\hline
\end{tabular}

performed during the 5-year study period and 95\% confidence intervals for the corresponding estimated 1-year survival. The donor risk indices shown were calculated using reference values of $8 \mathrm{~h}$ for cold ischemia time and local procurement for donor location. In Table 5, illustrative donor and transplant factors are sequentially altered beginning with the ideal organ, a whole liver from a donor with- out any risk factors for graft failure (donor risk index $\leq 1.0$ ), to show the impact of selected factors on the donor risk index.

\section{Adjusted graft survival according to donor risk index stratification}

Adjusted graft survival rates for various categories of the donor risk index are displayed in Table 6. The donor risk index calculation includes not only the seven identified donor factors but also the two identified transplant factors of regional/national sharing and cold ischemia time since these two variables are known or estimable at the time of organ offer. Graft survival differences between grafts with higher versus lower risk indexes are evident 3 months after transplantation and appear to increase over time. For instance, the $5.6 \%$ survival difference at 3 months for grafts with a donor risk index $\leq 1.0$ compared to grafts with a risk index between 1.5 and 1.6 increased to $7.7 \%$ at 1 year and $10.6 \%$ at 3 years.

\section{Recipient characteristics associated with utilization of grafts with higher donor risk index}

Linear regression was used to examine potential associations between the calculated donor risk index and various recipient characteristics. Data from 9153 recipients of deceased donor livers transplanted between April 1 , 2002 and December 31, 2003 were used in the regression model. Results are shown in Table 7. Compared to the reference recipient age group of 40-49, older recipients and the youngest recipients were significantly more likely to receive a graft associated with a higher donor risk index. For example, the donor risk index for organs transplanted into 60-69-year-old recipients was approximately 0.041 higher than that for organs transplanted into 40-49year-old recipients, corresponding to a $4.1 \%$ increase in the risk of graft failure $(p<0.0001)$. The donor risk index for livers transplanted into 0-10-year-old recipients was approximately 0.332 higher than for the reference recipient age group, corresponding to a $33.2 \%$ increase in the risk of graft failure $(p \leq 0.0001)$. Higher donor risk index organs were also transplanted into women and recipients without hepatitis $\mathrm{C}$. Although no differences in donor risk index

Table 5: Specific combinations of donor risk factors and the corresponding donor risk index

\begin{tabular}{|c|c|c|c|c|c|c|}
\hline Donor factor & Reference donor & Example 1 & Example 2 & Example 3 & Example 4 & Example 5 \\
\hline Age & Under 40 & 64 & 64 & 64 & 25 & 25 \\
\hline Cause of death & Trauma & Trauma & Stroke & Stroke & Trauma & Trauma \\
\hline Race & White & White & White & White & White & White \\
\hline DCD & No & No & No & No & No & Yes \\
\hline Partial/Split & No & No & No & No & No & No \\
\hline Height (cm) & 170 & 170 & 170 & 170 & 170 & 170 \\
\hline Location & Local & Local & Local & Local & Local & Local \\
\hline Cold time (h) & 8 & 8 & 8 & 14 & 14 & 14 \\
\hline Donor risk index* & 1.00 & 1.53 & 1.77 & 1.88 & 1.06 & 1.60 \\
\hline
\end{tabular}

*Calculation: Donor risk index $=\exp [(0.154$ if $40 \leq$ age $<50)+(0.274$ if $50 \leq$ age $<60)+(0.424$ if $60 \leq$ age $<70)+(0.501$ if $70 \leq$ age $)+$ $(0.079$ if $\mathrm{COD}=$ anoxia $)+(0.145$ if $\mathrm{COD}=\mathrm{CVA})+(0.184$ if $\mathrm{COD}=$ other $)+(0.176$ if race $=$ African American $)+(0.126$ if race $=$ other $)+$ $(0.411$ if DCD $)+(0.422$ if partial/split $)+(0.066((170$-height $) / 10))+(0.105$ if regional share $)+(0.244$ if national share $)+(0.010 \times$ cold time $)]$. 
Table 6: Adjusted 3-month, 1-year and 3-year graft survival according to donor risk index as determined by donor, graft and transplant factors (1998-2002)

\begin{tabular}{lllll}
\hline & & \multicolumn{3}{l}{ Graft survival (95\% confidence interval) } \\
\cline { 3 - 5 } Donor risk index & $\mathrm{N}(\%)$ & 3 Months & 1 Year & 3 Years \\
\hline $0.0<\mathrm{DRI} \leq 1.0$ & $3701(18.5)$ & $91.9(91.0-92.7)$ & $87.6(86.6-88.7)$ & $81.2(79.9-82.6)$ \\
$1.0<\mathrm{DRI} \leq 1.1$ & $2714(13.6)$ & $90.3(89.2-91.4)$ & $85.0(83.7-86.3)$ & $78.7(77.1-80.3)$ \\
$1.1<\mathrm{DRI} \leq 1.2$ & $2272(11.4)$ & $89.9(88.7-91.1)$ & $83.6(82.1-85.1)$ & $75.3(73.4-77.3)$ \\
$1.2<\mathrm{DRI} \leq 1.3$ & $1873(9.4)$ & $88.5(87.1-89.9)$ & $83.2(81.5-84.8)$ & $75.3(73.2-77.4)$ \\
$1.3<\mathrm{DRI} \leq 1.4$ & $1687(8.4)$ & $88.8(87.4-90.3)$ & $82.3(80.5-84.1)$ & $74.1(71.8-76.3)$ \\
$1.4<\mathrm{DRI} \leq 1.5$ & $1625(8.1)$ & $86.4(84.8-88.0)$ & $79.7(77.8-81.6)$ & $71.1(68.8-73.4)$ \\
$1.5<\mathrm{DRI} \leq 1.6$ & $1446(7.2)$ & $86.3(84.5-88.0)$ & $79.9(77.9-82.0)$ & $70.6(68.1-73.1)$ \\
$1.6<\mathrm{DRI} \leq 1.8$ & $2118(10.6)$ & $84.4(82.9-85.9)$ & $76.9(75.1-78.7)$ & $66.8(64.7-69.0)$ \\
$1.8<\mathrm{DRI} \leq 2.0$ & $1343(6.7)$ & $83.4(81.4-85.3)$ & $75.8(73.6-78.1)$ & $65.6(62.9-68.4)$ \\
$2.0<\mathrm{DRI}$ & $1244(6.2)$ & $80.3(78.1-82.6)$ & $71.4(68.8-74.1)$ & $60.0(56.9-63.2)$ \\
\hline
\end{tabular}

emerged by recipient medical condition, there were significant differences by recipient medical urgency. Grafts with higher donor risk index were most likely utilized for low disease severity (MELD score 10-14) recipients and least likely for Status 1 recipients.

\section{Liver disposition by donor risk index categories}

Table 8 shows discard rates for recovered livers from April 1, 2002 to December 31, 2003 by categories of donor risk index. Increasing donor risk index was associated with higher discard rates among deceased donor livers procured for transplant. Livers with a donor risk index $>1.5$ were discarded more than twice as often as those with a risk index $\leq 1.1$.

\section{Discussion}

We have identified seven donor and graft characteristics that are significantly and independently associated with increased failure of deceased donor liver transplants. It is deliberate and notable that the factors required to determine the relative risk associated with a particular graft are known at the time of organ offer. This enables transplant physicians to share information regarding the risk posed by any graft offer in juxtaposition to the candidate's disease severity at that moment. Such information can provide a rational foundation to discuss and decide the acceptability of any organ offer.

Among the donor and graft characteristics that were significantly associated with liver graft failure; age; DCD and split/partial status dominated over donor race, height and cause of brain death. The relative risk associated with each decade of increasing donor age rose steeply, beginning at 40 years. The importance of donor age as a negative factor is accentuated by the soaring frequency of older donors. While livers from donors greater than 40 years accounted for about $13 \%$ of adult transplants in 1988, they accounted for $54 \%$ of adult transplants in 2003. Since the strong negative impact of increased donor age on liver transplant outcomes has long been recognized (8-12), the dramatic in- crease in utilization of livers from older donors reflects the increasing disparity between organ demand and supply.

Similarly, transplantation of split/partial or DCD grafts is associated with greater than $50 \%$ increased risk of graft failure compared to transplantation of whole or donation after brain death grafts, respectively. Although DCD and split/partial status are qualitatively different than the other donor characteristics, they were included to provide a more comprehensive description of risk according to donor and graft characteristics. Currently, split/partial and DCD liver transplants account for only $2.0 \%$ and $1.1 \%$ of all transplants, respectively. The numbers of these transplants has and will likely continue to increase (13). Therefore, risk assessment for these grafts will be increasingly useful for physician and patient decision making.

There is a substantial body of literature presenting algorithms to assess the risk of graft failure after liver transplantation. While there are some similarities between our current analysis and previous reports, there are some notable differences that warrant discussion. The majority of published studies have examined donor, recipient and/or transplant factors (14-22). In contrast, our study focused on donor characteristics while adjusting for an extensive list of important recipient and transplant characteristics. Several donor characteristics previously identified by others as risk factors did not achieve significance in our analysis. These included female sex, obesity, elevated liver function tests (aminotransferases), hypotension/increased pressor use and elevated levels of serum sodium. Two other factors, macrosteatosis and cold ischemia time, have been strongly associated with transplant outcomes $(19,23$ 27). The degree of macrosteatosis, which may or may not be known at the time of organ offer, was not significantly associated with graft failure in our study. Estimation of steatosis using frozen section liver biopsy is both difficult and subjective $(28,29)$. Moreover, OPTN data regarding steatosis are recorded in broad ranges and, until recently, did not differentiate between macro- and micro-steatosis. Therefore, insufficient data may in part explain the lack of association between macrosteatosis and graft failure. In 
Table 7: Donor risk index according to recipient characteristics for transplants between April 2002 and December 2003

\begin{tabular}{|c|c|c|c|c|}
\hline \multirow{2}{*}{$\begin{array}{l}\text { Recipient } \\
\text { parameter }\end{array}$} & \multirow{2}{*}{$\begin{array}{l}\text { Difference } \\
\text { from } \\
\text { reference } \\
\text { group }\end{array}$} & \multirow[b]{2}{*}{$p$-Value } & \multicolumn{2}{|c|}{$\begin{array}{l}95 \% \text { Confidence } \\
\text { limits for donor }\end{array}$} \\
\hline & & & Upper & Lower \\
\hline \multicolumn{5}{|l|}{ Recipient age } \\
\hline $0-10$ & 0.332 & $<.0001$ & 1.65 & 1.74 \\
\hline $11-17$ & -0.052 & 0.024 & 1.24 & 1.34 \\
\hline $18-39$ & -0.012 & 0.362 & 1.30 & 1.36 \\
\hline $40-49$ & 1.343 & Ref. & & \\
\hline $50-59$ & 0.033 & $<0.0001$ & 1.36 & 1.40 \\
\hline $60-69$ & 0.041 & $<0.0001$ & 1.36 & 1.41 \\
\hline $70+$ & 0.116 & $<0.0001$ & 1.41 & 1.52 \\
\hline \multicolumn{5}{|l|}{ Race } \\
\hline Black & -0.010 & 0.385 & 1.35 & 1.39 \\
\hline White & 1.380 & Ref. & & \\
\hline Other & 0.039 & 0.004 & 1.39 & 1.45 \\
\hline \multicolumn{5}{|l|}{ Sex } \\
\hline Male & -0.041 & $<.0001$ & 1.35 & 1.38 \\
\hline Female & 1.408 & Ref. & & \\
\hline \multicolumn{5}{|l|}{ Diagnosis } \\
\hline $\mathrm{AHN}$ & -0.011 & 0.427 & 1.34 & 1.40 \\
\hline $\begin{array}{c}\text { Non-cholestatic } \\
\text { liver disease }\end{array}$ & 1.381 & Ref. & & \\
\hline Cholestatic & -0.009 & 0.437 & 1.35 & 1.40 \\
\hline liver disease & & & 1.35 & 1.40 \\
\hline Metabolic disease & -0.023 & 0.224 & 1.32 & 1.40 \\
\hline Malignant neoplasms & 0.000 & 0.990 & 1.35 & 1.41 \\
\hline Other & 0.023 & 0.047 & 1.38 & 1.43 \\
\hline \multicolumn{5}{|l|}{ Hepatitis C } \\
\hline Positive & -0.027 & 0.001 & 1.35 & 1.38 \\
\hline Negative & 1.391 & Ref. & & \\
\hline \multicolumn{5}{|l|}{ Medical condition } \\
\hline Not in hospital & 1.377 & Ref. & & \\
\hline Missing & 0.008 & 0.894 & 1.26 & 1.52 \\
\hline In hospital & 0.018 & 0.069 & 1.38 & 1.42 \\
\hline $\ln I C U$ & 0.008 & 0.536 & 1.36 & 1.41 \\
\hline \multicolumn{5}{|l|}{ Medical urgency } \\
\hline Status 1 & -0.058 & 0.007 & 1.29 & 1.38 \\
\hline MELD $<10$ & 1.399 & Ref. & & \\
\hline MELD 10-14 & 0.044 & 0.026 & 1.40 & 1.49 \\
\hline MELD 15-19 & 0.008 & 0.657 & 1.37 & 1.45 \\
\hline MELD 20-24 & -0.020 & 0.256 & 1.34 & 1.41 \\
\hline MELD 25-29 & -0.031 & 0.090 & 1.33 & 1.40 \\
\hline MELD 30-34 & -0.026 & 0.180 & 1.33 & 1.41 \\
\hline$M E L D \geq 35$ & -0.044 & 0.027 & 1.31 & 1.39 \\
\hline
\end{tabular}

contrast, cold ischemia time, which was associated with risk of graft failure and which can be reasonably estimated at the time of organ offer, was included in our donor risk index along with the information about organ sharing at the regional or national level.

Our study provides a risk assessment for every potential liver graft compared to the ideal liver graft (i.e., a whole organ from a donor less than age 40 with brain death secondary to trauma or anoxia). While ideal grafts are a relatively homogeneous group, non-ideal grafts are quite heterogeneous, spanning a broad continuum of graft failure risk. The heterogeneity reflects the variable impact of not only individual donor and recipient risk factors but also the multitude of possible risk factor combinations presented by the donor pool. Given prevailing trends, the risk of failure of the average donor liver will likely continue to increase unless advances in organ preservation, utilization and/or implantation techniques attenuate the negative impact exerted by one or more risk factors. The age of the average donor is rising, as is the frequency of DCD and split or partial liver grafts (13). Nearly one-third of all deceased liver donors in 2003 were over age 50. Over the past 5 years, the number of split or partial liver grafts has increased by $35 \%$, and the number of livers from DCD donors has quadrupled. It is ironic that the majority of livers that are split (a significant risk factor for graft failure) come from donors that would have been considered ideal if transplanted as a whole organ (30-32). However, although the individual outcomes for adult recipients of split grafts are inferior to those of whole grafts, splitting improves societal benefit by increasing both the number of patients transplanted and the net gain of life years (33).

The characteristics of typical recipients of higher risk grafts presumably reflect the balanced choice that transplant physicians have made in an attempt to maximize candidate benefit. Candidates who are most ill face the greatest risk of death without transplantation and have the greatest survival benefit from transplantation $(34,35)$. However, candidates who are most ill may have disproportionately poorer outcomes with higher risk grafts, although the interaction between donor quality and recipient disease severity is as yet incompletely defined. Amin and colleagues published the results of a Markovian model to elucidate the risk and benefit considerations for accepting or declining a liver offer according to the organ's potential for failure and the candidate's disease severity, as specified by MELD (36). Using probabilistic criteria to define an expanded criteria graft based on primary graft failure risk, they compared immediate transplantation with such livers to delayed transplantation using a 'standard' donor organ. Immediate transplantation with grafts bearing as much as a $50 \%$ risk of primary graft failure resulted in higher 1-year survival for any candidate whose MELD score exceeded 20 compared to waiting; the magnitude of benefit increased as MELD score increased. While some of the assumptions of the analysis may be called into question-including the possibility of recovery from primary graft failure, the rate of retransplantation for primary graft failure, the lack of consideration of late graft failure and the availability of a standard criteria donor liver-the 1-year survival benefit afforded by immediate transplantation using higher risk organs was indeed striking. To validate the concept of the donor risk index, an extension of our current work is planned, in which we will formally examine the interaction between donor risk index and recipient risk factors, using national data instead of a modeling approach.

As expected, discard rates for organs with higher donor risk index are higher than for those with lower risk index. 
Table 8: Disposition of 9882 deceased donor livers recovered for transplant by donor risk index (April 1, 2002 to December 31, 2003)

\begin{tabular}{lllll}
\hline Donor risk index* & Recovered, N & Transplanted, N (\%) & Discarded, N (\%) & p-Value \\
\hline $0.0-1.0$ & 1972 & $1910(96.9)$ & $62(3.1)$ & Ref. \\
$1.0-1.1$ & 1202 & $1153(95.9)$ & $49(4.1)$ & 0.1672 \\
$1.1-1.2$ & 1006 & $943(93.7)$ & $63(6.3)$ & $<0.0001$ \\
$1.2-1.3$ & 832 & $782(94.0)$ & $50(6.0)$ & 0.0005 \\
$1.3-1.4$ & 876 & $785(89.6)$ & $91(10.4)$ & $<0.0001$ \\
$1.4-1.5$ & 842 & $762(90.5)$ & $80(9.5)$ & $<0.0001$ \\
$1.5-1.6$ & 752 & $673(89.5)$ & $79(10.5)$ & $<0.0001$ \\
$1.6-1.8$ & 1174 & $1056(89.9)$ & $118(10.1)$ & $<0.0001$ \\
$1.8-2.0$ & 722 & $648(89.8)$ & $74(10.2)$ & $<0.0001$ \\
$2.0+$ & 504 & $441(87.5)$ & $63(12.5)$ & $<0.0001$ \\
Total & 9882 & $9153(92.6)$ & $729(7.4)$ & \\
\hline
\end{tabular}

*Donors are considered to be in the local donor service area with $8 \mathrm{~h}$ of cold ischemia time, since these factors are unknown for discarded organs.

However, compared to the discard rate of $38 \%$ reported for procured expanded criteria donor kidneys (2), the discard rate for higher donor risk index organs in our study was much more modest, exhibiting an increasing trend from $3.1 \%$ for organs procured from donors with a risk index of 1.0 to a maximum of $12.5 \%$ for those procured from a donor with a risk index of 2.0 or greater. This difference may in part reflect a greater willingness on the part of transplant physicians and candidates to accept increased risk from suboptimal donor quality in the face of more imminent considerations of candidate mortality in the absence of transplantation. Moreover, the intended liver recipient is almost invariably identified prior to procurement, while deceased donor kidneys are often procured before a recipient has agreed to accept the organ. Practice patterns of organ procurement, utilization and discard may shift toward increasing use of higher risk liver grafts as candidates with higher risk profiles are considered and accepted for transplantation.

Ultimately, at the time of an organ offer, the decision to accept either the risk of transplantation or the risk of waiting rests with transplant physicians and their patients. Making this decision rationally, however, requires facts about the risk posed by the particular graft being offered and the risk of death from progressive liver disease if the current offer is declined. Since the quality of the donor organ is such an important component of this decision, our study provides an important quantitative assessment of relative risk for every potential graft, given the characteristics of the intended recipient, based upon donor and graft characteristics available at the time the organ is offered. This information is necessary to inform discussions of organ acceptance, both in general terms with patients and their families, and at the time an offer is made.

\section{Acknowledgment}

The Scientific Registry of Transplant Recipients is funded by contract number 231-00-0116 from the Health Resources and Services Administration, U.S. Department of Health and Human Services. The views expressed herein are those of the authors and not necessarily those of the U.S. Government.

\section{References}

1. Busuttil RW, Tanaka K. The utility of marginal donors in liver transplantation. Liver Transpl 2003; 9: 651-663.

2. Port FK, Bragg-Gresham JL, Metzger RA et al. Donor characteristics associated with reduced graft survival: an approach to expanding the pool of kidney donors. Transplantation 2002; 74: $1281-1286$.

3. Botta F, Giannini E, Romagnoli P et al. MELD scoring system is useful for predicting prognosis in patients with liver cirrhosis and is correlated with residual liver function: a European study. Gut 2003; 52: 134-139.

4. Kamath PS, Wiesner $\mathrm{RH}$, Malinchoc $\mathrm{M}$ et al. A model to predict survival in patients with end-stage liver disease. Hepatology 2001; 33: 464-470.

5. Wiesner R, Edwards E, Freeman R et al. Model for end-stage liver disease (MELD) and allocation of donor livers. Gastroenterology 2003; 124: 91-96.

6. Wolfe RA, Schaubel DE, Webb RL et al. Analytical approaches for transplant research. Am J Transplant 2004; 4(Suppl 9): 106-113.

7. Allison PD. Missing data. Quantitative Applications in the Social Sciences. Volume 136. Thousand Oaks CA: Sage Publications; 2002.

8. Detre KM, Lombardero M, Belle S et al. Influence of donor age on graft survival after liver transplantation-United Network for Organ Sharing Registry. Liver Transpl Surg 1995; 1: 311-319.

9. Yersiz $H$, Shaked A, Olthoff $K$ et al. Correlation between donor age and the pattern of liver graft recovery after transplantation. Transplantation 1995; 60: 790-794.

10. Hoofnagle JH, Lombardero M, Zetterman RK et al. Donor age and outcome of liver transplantation. Hepatology 1996; 24: 89-96.

11. Oh CK, Sanfey HA, Pelletier SJ, Sawyer RG, McCullough CS, Pruett TL. Implication of advanced donor age on the outcome of liver transplantation. Clin Transplant 2000; 14(4 Pt 2): 386-390.

12. Busquets $J, X i o l X$, Figueras $J$ et al. The impact of donor age on liver transplantation: influence of donor age on early liver function and on subsequent patient and graft survival. Transplantation 2001; 71: 1765-1771.

13. 2004 Annual Report of the U.S. Organ Procurement and Transplantation Network and the Scientific Registry of Transplant Recipients: Transplant Data 1994-2003. Department of Health and 
Human Services, Health Resources and Services Administration, Special Programs Bureau, Division of Transplantation, Rockville, MD; United Network for Organ Sharing, Richmond, VA; University Renal Research and Education Association, Ann Arbor, MI. 2004.

14. Gruenberger T, Steininger R, Sautner T, Mittlbock M, Muhlbacher F. Influence of donor criteria on postoperative graft function after orthotopic liver transplantation. Transpl Int 1994; 7(Suppl 1): S672S674.

15. Gonzalez FX, Rimola A, Grande L et al. Predictive factors of early postoperative graft function in human liver transplantation. Hepatology 1994; 20: 565-573.

16. Strasberg SM, Howard TK, Molmenti EP, Hertl M. Selecting the donor liver: risk factors for poor function after orthotopic liver transplantation. Hepatology 1994; 20(4 Pt 1): 829-838.

17. Briceno J, Solorzano G, Pera C. A proposal for scoring marginal liver grafts. Transpl Int 2000;13(Suppl 1): S249- S252.

18. Markmann JF, Markmann JW, Markmann DA et al. Preoperative factors associated with outcome and their impact on resource use in 1148 consecutive primary liver transplants. Transplantation 2001; 72: 1113-1122.

19. Zamboni F, Franchello A, David E et al. Effect of macrovescicular steatosis and other donor and recipient characteristics on the outcome of liver transplantation. Clin Transplant 2001; 15: 53-57.

20. Ghobrial RM, Gornbein J, Steadman R et al. Pretransplant model to predict posttransplant survival in liver transplant patients. Ann Surg 2002; 236: 315-322; discussion 322-323.

21. Bilbao I, Armadans L, Lazaro JL, Hidalgo E, Castells L, Margarit C. Predictive factors for early mortality following liver transplantation. Clin Transplant 2003; 17: 401-411.

22. Rull R, Vidal O, Momblan D et al. Evaluation of potential liver donors: limits imposed by donor variables in liver transplantation. Liver Transpl 2003; 9: 389-393.

23. Furukawa $\mathrm{H}$, Todo $\mathrm{S}$, Imventarza $\mathrm{O}$ et al. Effect of cold ischemia time on the early outcome of human hepatic allografts preserved with UW solution. Transplantation 1991; 51: 1000-1004.

24. Marsman WA, Wiesner $\mathrm{RH}$, Rodriguez $\mathrm{L}$ et al. Use of fatty donor liver is associated with diminished early patient and graft survival. Transplantation 1996; 62: 1246-1251.
25. Selzner M, Clavien PA. Fatty liver in liver transplantation and surgery. Semin Liver Dis 2001; 21: 105-113.

26. Verran $D$, Kusyk $T$, Painter $D$ et al. Clinical experience gained from the use of 120 steatotic donor livers for orthotopic liver transplantation. Liver Transpl 2003; 9(5): 500-505.

27. Totsukali E, Fung JJ, Ishizawa Y et al. Synergistic effect of cold and warm ischemia time on postoperative graft outcome in human liver transplantation. Hepatogastroenterology 2004; 51: 14131416.

28. Franzen LE, Ekstedt M, Kechagias S, Bodin L. Semiquantitative evaluation overestimates the degree of steatosis in liver biopsies: a comparison to stereological point counting. Mod Pathol 2005;18: 912-916.

29. Fiorini RN, Kirtz J, Periyasamy B et al. Development of an unbiased method for the estimation of liver steatosis. Clin Transplant 2004; 18: 700-706.

30. Ghobrial RM, Yersiz H, Farmer DG et al. Predictors of survival after In vivo split liver transplantation: analysis of 110 consecutive patients. Ann Surg 2000; 232: 312-323.

31. Azoulay D, Castaing D, Adam R et al. Split-liver transplantation for two adult recipients: feasibility and long-term outcomes. Ann Surg 2001; 233: 565-574.

32. Humar A, Ramcharan T, Sielaff TD et al. Split liver transplantation for two adult recipients: an initial experience. Am J Transplant 2001; 1: 366-372.

33. Merion RM, Rush SH, Dykstra DM Goodrich N, Freeman RB, Jr, Wolfe RA. Predicted lifetimes for adult and pediatric split liver versus adult whole liver transplant recipients. Am J Transplant 2004; 4: 1792-1797.

34. Merion RM. When is a patient too well and when is a patient too sick for a liver transplant? Liver Transpl 2004;10(10 Suppl 2): S69-S73.

35. Merion RM, Schaubel DE, Dykstra DM et al. The survival benefit of liver transplantation. Am J Transplant 2005; 5: 307313.

36. Amin MG, Wolf MP, TenBrook JA, Jr et al. Expanded criteria donor grafts for deceased donor liver transplantation under the MELD system: a decision analysis. Liver Transpl 2004; 10: 14681475. 